\title{
Spinal Cord Protection During Open and Endovascular Aortic Repair: State of the Art
}

\author{
Carlo Setacci, Marco Tadiello, Matteo Tozzi, \\ Giancarlo Palasciano, Domenico Benevento, \\ Gianmarco de Donato, and Francesco Setacci
}

\subsection{Introduction}

Spinal cord protection during aortic surgery has spelled trouble since the beginning of aortic surgery.

As reported by Clopton in 1911 [1], A. Carrel said: "The main danger of the aortic operation does not come from the heart or from the aorta itself, but from the central nervous system. Spastic paralysis develops when the descending aorta has been clamped for more than ten or fifteen minutes due to a degeneration of the cells in the anterior horn".

C. Setacci $(\bowtie) \cdot$ M. Tadiello $\cdot$ G. Palasciano

D. Benevento · G. de Donato

Vascular and Endovascular Surgery Unit,

Department of Medicine, Surgery and Neurological

Sciences, University of Siena, Siena, Italy

e-mail: carlo.setacci@unisi.it;

marco.tadiello@student.unisi.it;

giancarlo.palasciano@unisi.it;

d.benevento@ao-siena.toscana.it;

gianmarco.dedonato@unisi.it

M. Tozzi

Vascular Surgery, Department of Medicine and

Surgery, Circolo University Teaching Hospital,

University of Insubria School of Medicine,

Varese, Italy

e-mail: matteo.tozzi@uninsubria.it

F. Setacci

Vascular Surgery Unit, Multimedica Institute for

Scientific Research, Milan, Italy

e-mail: francesco.setacci@multimedica.it
A hundred years later, spinal cord protection during aortic repair still remains a topic of major debate.

The incidence of paraplegia was reported to be as high as $20 \%$ in the early 1990s [2], and current scientific literature describes spinal cord ischaemia (SCI) as the most dreaded complication of both open and endovascular repair of thoracoabdominal aortic disease, with reported rates between 5 and $31 \%$ [3-7].

\subsubsection{Anatomy of the Spinal Cord Blood Supply}

Understanding the spinal cord (SC) blood supply is a cornerstone when treating patients requiring thoracic and thoracoabdominal aortic repair. The SC vessel network is complex and difficult to study because it consists of very small and intricate vessels with substantial regional and interindividual variability. Most of our anatomical knowledge about SC circulation derives from microinjections of different staining fluids and micro-radiological studies in postmortem specimens [8].

The extrinsic arterial system, supplying inflow vessels to the $\mathrm{SC}$, is constituted by the subclavian artery through the vertebral artery, the thyrocervical trunk and the costocervical trunk, several segmental feeders from the intercostal and lumbar arteries and the hypogastric artery through the lateral sacral and iliolumbar arteries [9]. 
The intrinsic arterial system, directly supplying the $\mathrm{SC}$, is divided into a central system (supplying blood to the spinal grey matter) fed by the sulcal arteries, arising from the anterior spinal artery (ASA), and a peripheral system, the pial plexus (or pial network), which gives rise to perforating branches. The pial plexus covers the entire length of the SC and forms an elaborate secondary anastomotic system between the anterior and posterolateral longitudinal vessels that may only be important for slower circulatory adjustments. Intraparenchymal anastomoses have rarely been demonstrated.

The intercostal arteries divide three times before reaching the ASA: the first branch of the intercostal artery is the nervomedullary artery, the latter divides into an anterior and a posterior radicular artery, and the anterior radicular artery divides into a descending and an ascending branch.

The ASA is essentially an anastomotic channel between ascending and descending branches of neighbouring anterior radicular arteries.

In the thoracolumbar region, one (occasionally two or three) anterior radicular artery (the artery of Adamkiewicz) is always distinctly dominant in calibre and is therefore called the great radicular artery or ARM [10].

The importance of the ARM was early described by A. Adamkiewicz in 1881-1882 [11]. Adamkiewicz demonstrated that blood from the radicular arteries flows upwards in the ascending branches and downwards in the descending ones. He also pointed out that the ARM is characterized by the most stable morphology and course. In $60 \%$ of the population it branches off at the level of the ninth to twelfth thoracic segment; in $15 \%$, at the level of the fifth to eighth segment; and in approximately $25 \%$, at the first lumbar segment [12].

The majority of Adamkiewicz's observations are fully confirmed by today's knowledge of the variability of the spinal cord vascular system and origin of the ARM.

Some peculiar anatomical variant types can hide the highest risk of paraplegia. Fortunately, in most rare anatomical variants with poor development of the ARM or a lack of continuity of the anterior spinal artery (i.e. it is interrupted in the majority of cases in its thoracic segment), clamping the thoracic aorta in the course of surgery, or covering a certain region with endografts, is associated with the highest risk of paraplegia [13].

\subsubsection{Imaging for Spinal Cord Protection}

The importance of preoperative knowledge of the location of the artery of Adamkiewicz (arteria radicularis magna, ARM) before thoracic and thoracoabdominal aortic surgery was demonstrated by Kieffer and co-workers back in the 1980s [14], and its clinical importance in both open and in endovascular aortic repair has been widely confirmed in more recent times for the prevention of spinal ischaemia $[2,15,16]$.

In open surgery, the precise preoperative location of ARM contributes to the reduction of the incidence of spinal cord ischaemia in thoracic aortic disease, shortening clamp time by identifying intercostal arteries that need to be reimplanted [17]. In endovascular aortic repair, it may define the most appropriate landing zone, avoiding unnecessary coverage of important intercostal arteries [18].

The rapid evolution of digital imaging techniques and the increasing number of multidimensional and multimodality studies constitute a challenge for picture archiving and communication system (PACS) workstations and image display programs. Traditional 2D image viewers and image display programs have become inadequate for interpretation of large sets of images. The large sets of images acquired by multidetector CT scanners require additional image processing and reformatting to make them suitable for efficient and rapid image navigation and interpretation. In most cases, this can only be achieved on high-end dedicated workstations that provide multiplanar/centre line reconstruction and 3D volume surface. Access to these techniques is usually limited to high-end users in a radiology 


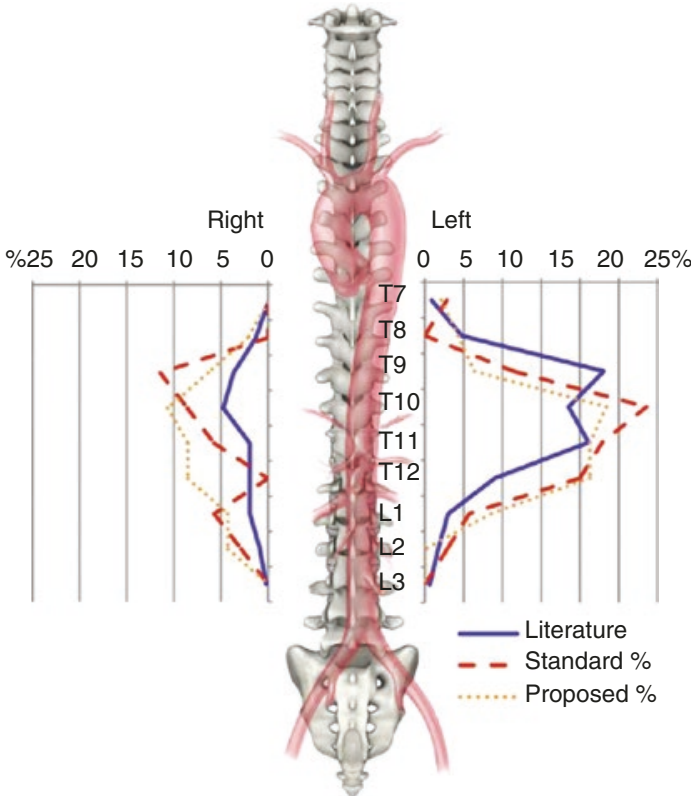

Fig. 27.1 Percentage of ARM origin level in relation to the intercostal artery, its location and method used. Note the higher left T9-T12 prevalence (G. Melissano, L. Bertoglio, V. Civelli, A.C. Moraes Amato, G. Coppi, E. Civilini, G. Calori, F. De Cobelli, A. Del Maschio, R. Chiesa, Demonstration of the Adamkiewicz Artery by Multidetector Computed Tomography Angiography Analysed with the Open-Source Software OsiriX. Eur J Vasc Endovasc Surg (2009) 37, 395e400) department, but since the last decade, many attractive alternatives to dedicated workstations have been made available. The most popular and easy to use is the software OsiriX, developed 8 years ago by Ratib and Rosset at the Department of Medical Imaging and Information Sciences at the University Hospital of Geneva, Switzerland [19].

A recent study by Melissano et al. [20] proposed a method for the demonstration of the ARM using OsiriX software.

In this study, the proposed method for ARM identification, using OsiriX software, had a substantial recognition consensus of $85.07 \%$ compared to the standard method, using a dedicated workstation and when applied to the same patients. The proposed method presents a higher prevalence of left (T9-T12) ARM arteries (Fig. 27.1) that is consistent with the standard method and the analysis of the literature.

In employing this versatile tool, they realized that identification of the ARM could be quite simple from a standard set of contrast-enhanced CT scans, showing the feasibility of ARM location by using low-cost hardware and software, with standard contrast protocol [20] (Fig. 27.2).
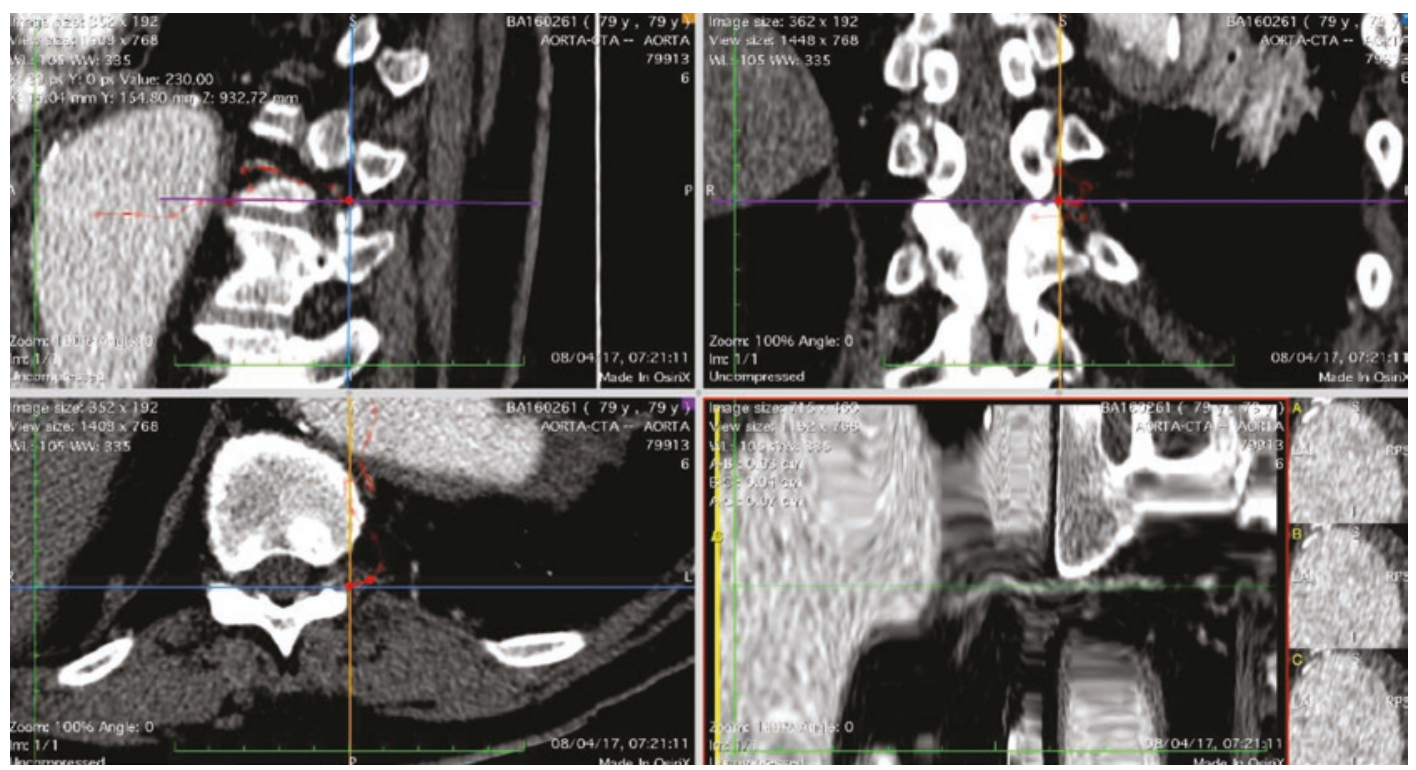

Fig. 27.2 Spinal artery multiplanar rendering using OsiriX software (Courtesy of M. Bossi, MD, HSR, Milan) 


\subsection{Mechanism of Spinal Cord Ischaemia}

Spinal cord ischaemia can develop basically after coverage/exclusion of intercostal arteries or after small thrombus fragment embolization.

Data from the EuREC (European Registry of Endovascular Aortic Repair Complications) suggest that extensive coverage of intercostal arteries by a thoracic stent graft alone is not associated with symptomatic spinal cord ischaemia, as the sacrifice of one vascular territory supplying the spinal cord is irrelevant. Simultaneous closure of at least two vascular territories instead is highly relevant, especially in combination with prolonged intraoperative hypotension.

By regarding all four vascular territories supplying the spinal cord-left subclavian, intercostal, lumbar and hypogastric arteries-as equally important, risk modelling found strong associations between simultaneous coverage of two of these independent vascular territories, intraoperative prolonged hypotension and the occurrence of symptomatic spinal cord injury.

It is well known how the preservation of the left subclavian artery, not because of left upper extremity perfusion, as this is always well maintained by collateral circulation, but to maintain the important perfusion pathway for the spinal cord, results in a low rate of paraplegia after TEVAR.

Interestingly, occlusion of one hypogastric artery during vascular access surgery was of equal importance as was the occlusion of the left subclavian artery [21].

The same notable remark derives from M. Eagleton's work (Cleveland Clinic, $\mathrm{OH}$ ) with the observation that extensive coverage of intercostal arteries by TEVAR alone was not associated with symptomatic SCI, as the sacrifice of one collateral bed was irrelevant. In particular, he also suggested that efforts to reduce the untoward effects of spinal cord malperfusion may be achieved by preserving flow (or restoring flow) to occluded subclavian and hypogastric collaterals prior to extensive endovascular aortic coverage [22].

\subsection{Spinal Cord Monitoring}

The assessment of spinal cord vascularization during surgery is a difficult goal to achieve due to the anatomical position of the spine and its extremely complex vessel net. Indirect techniques can however assess the SC vitality and can lead the surgeon's decisions during either open or endovascular procedures and in the immediate postoperative period.

\subsubsection{Cerebrospinal Fluid Drainage}

The choroid plexus and walls of the ventricles produce cerebrospinal fluid at a rate that varies in a diurnal rhythm from 0.2 to $0.7 \mathrm{~mL} / \mathrm{min}$, producing 400-600 $\mathrm{mL}$ of fluid every $24 \mathrm{~h}$. Cerebrospinal fluid circulates from the brain to the spinal canal and back to the brain where it is reabsorbed in the venous sinuses. The total volume of spinal circulating fluid is approximately $140 \mathrm{~mL}$ in an adult, and the ventricles hold an additional $25 \mathrm{~mL}$. Spinal cord perfusion pressure derives from the difference between arterial blood pressure and SCF pressure. When SCF pressure exceeds spinal arterial pressure, a critical closing pressure is achieved [23].

The relationship between cerebrospinal fluid pressure and the development of paraplegia during repairs of the thoracic aorta was first suggested by Sugie and colleagues in 1957 [24].

Almost three decades later, experimental work by McCullough and colleagues [25] and Bower and colleagues [26] at the Mayo clinic led to reports on the benefit of perioperative cerebrospinal fluid drainage (CSFD) and renewed interest in the use of CSFD for thoracic aortic repairs.

Nowadays, hundreds of clinical studies focus on CSFD, and the protocol for its management is quite different from one team to another: intraoperative or both intra- and postoperative, at 24-72 $\mathrm{h}$ postoperatively, maintaining cerebrospinal fluid pressure at 5 or $10 \mathrm{mmHg}$ (or $\mathrm{cm} \mathrm{H} 2 \mathrm{O}$ ) and systematic drainage of $50 \mathrm{~mL}$ or more.

An example of the current policies is the one of King's College London, UK. Their protocol is to place a spinal drain prior to surgery for all Crawford type I, II, III and V TAAAs and to begin 
prophylactic CSF drainage intraoperatively. Passive drainage of CSF is done at $10 \mathrm{~cm} \mathrm{H} 2 \mathrm{O}$ until postoperative day 2 , during which time all patients are monitored hourly for the presence of lower limb neurology. If any signs or symptoms of spinal cord ischaemia develop, the MAP is raised to $>90$ to $100 \mathrm{mmHg}$ using inotropic support and the CSF drain initially lowered to $5 \mathrm{~cm}$ $\mathrm{H} 2 \mathrm{O}$, maintaining drainage for up to 5 days [6].

In order to avoid many practical disadvantages of the current gravity-based drip chamber system, some automatic drainage systems are appearing. The most remarkable drawbacks of the current systems are the impossibility of a simultaneous drainage and pressure measurement and possible handling mistakes that are related to concerns of safety, sterility and human error, leading to potentially dangerous over-/underdrainage and catheter contamination.

The San Raffaele group (Y. Tshomba et al.) recently utilized an automatic drainage system (the LiquoGuard (Möller Medical $\mathrm{GmbH}$, Fulda, Germany)). This device allows CSF drainage according to CSF pressure changes. The pressure sensor, along the tubing, continuously measures the pressure in the CSF catheter, and the volume of CSF pumped out is also continuously measured. The CSF automatic drainage system has the advantage that the CSF is drained not simply on the basis of the excess pressure but is actively pumped out in a controlled manner, with the possibility of controlling hourly drainage speed, maximum and minimum CSF pressure and maximum amount to be drained per hour.

Apart from the previously mentioned advantages, they observed a reduced post-dural puncture headache with earlier patient mobilization and better compliance [27].

\subsubsection{Neuromonitoring}

Since the early work of Coles and colleagues [28] and Cunnigam and colleagues [29, 30] in 1982, demonstrating that it was possible to detect spinal cord ischaemia intraoperatively by monitoring somatosensory evoked potential (SSEPs), grow- ing interest has spread among vascular surgeons in applying this technique to TAAA surgery.

Nowadays, motor evoked potentials (MEPs) enable the surgeon to better understand the ischaemic damage of the spinal cord.

From a physio-anatomical point of view, SSEPs are the expression of the spinal grey matter dorsal column vitality, while MEPs are the expression of the anterolateral ones.

In brief, electrical charges sent to the motor cortex through $\mathrm{C} 3 / \mathrm{C} 4$ scalp electrodes evoke MEPs through the motor pathway, and values are recorded down the cord over multiple muscles in the upper and lower extremities. An upper extremity muscle is recorded to help differentiate neurogenic impairment from nonspecific changes. Bilateral lower extremity muscles are recorded using subdermal electroencephalographic (EEG) electrodes generally placed in the hamstring, tibialis anterior and abductor hallucis muscles. In addition, SSEPs, consisting of electrical stimulus generated at the ulnar or tibial nerve that travels from the distal extremity, are recorded over the neck and scalp. A $\geq 75 \%$ consistent reduction from baseline evoked potential amplitude is generally considered to be significant (Fig. 27.3).

The main goal of neuromonitoring is to help identify ischaemia of the anterior and lateral spinal columns, which are sensitive to a decrease in oxygen supply. Because signal changes can result from dysfunction in any of the recorded pathways, including the brain, peripheral nerves, receptors or muscles, as well as technical issues, interpretation requires correlation with the clinical scenario.

During endovascular procedures, early detection of these changes allows the surgical team to put in place manoeuvres designed to optimize spinal cord perfusion pressure (augmentation of MAP, reduction of CSF pressure, early restoration of pelvis and lower limb flow) in order to reduce the risk of spinal cord injury.

During open TAAA repair, this technique has helped guide selective intercostal artery reimplantation and the aforementioned intraoperative manoeuvres designed to improve spinal cord perfusion [31]. 


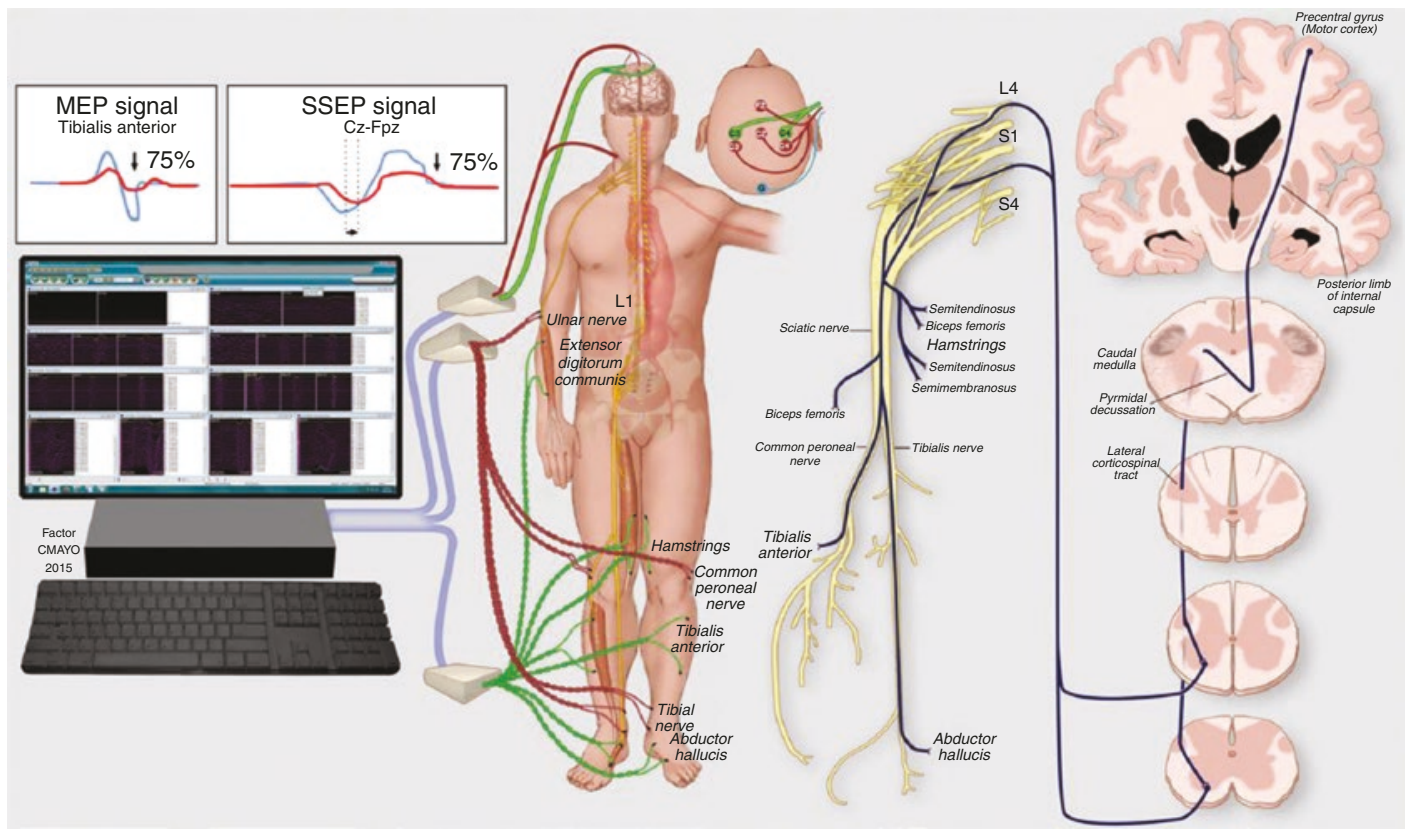

Fig. 27.3 Illustration depicting the placement of electrodes for monitoring of motor evoked potentials (MEPs) and somatosensory evoked potentials (SSEPs) during complex endovascular aortic repair. A significant change is defined by $>75 \%$ decline in amplitude in MEPs or SSEPs (Peter V. Banga, Gustavo S. Oderich, Leonardo Reis de Souza, Jan Hofer, Meaghan L. Cazares Gonzalez,

\subsection{Operative Strategies for Preventing SCI in Endovascular Aortic Repair}

Following endovascular aortic procedures, SCI can occur from a combination of reduced spinal cord perfusion, because of hypotension, coverage of lumbar and intercostal arteries and/or embolization of atheroma/thrombus into the aforementioned.

If the risk of thrombus mobilization and embolization during endovascular manoeuvers remains a less predictable inconvenience, the "haemodynamic" causes of SC ischaemia are a foreseeable one. Careful management of the arterial pressure during the intervention is mandatory. The preservation of the left subclavian/ hypogastric arteries, in cases of favourable anatomy, in order to maintain the collateral extrinsic $\mathrm{SC}$ vasculature is at present a solid knowledge;
Juan N. Pulido, Stephen Cha, Peter Gloviczki, Neuromonitoring, Cerebrospinal Fluid Drainage, and Selective Use of Iliofemoral Conduits to Minimize Risk of Spinal Cord Injury During Complex Endovascular Aortic Repair, Journal of Endovascular Therapy 2016, Vol. 23(1) 139-149)

in addition, the early removal of large sheets and the selective use of iliofemoral conduit are proved to be solid adjuncts in order to prevent $\mathrm{SC}$ ischaemia.

The deepest consciousness of the spinal cord vasculature acquired with the aforementioned diagnostic tools and the so-derived collateral network concept allows the clinician to put in place several methods to stimulate the spinal cord collateral network in order to avoid the onset of ischaemia.

\subsubsection{Staged Endovascular Repair and Temporary Aneurysm Sac Perfusion}

Early reports of successful thoracoabdominal aneurysm repair suggested that postoperative neurologic dysfunction occurred in proportion to the extent of aortic replacement [2]. The endo- 
vascular approach to the aorta allows minimal surgical injury and the absence of organ ischaemia, but it does require the coverage of any patent intercostals and/or lumbar vessels that may contribute to spinal cord blood flow suffering from the inability to maintain their direct perfusion. Aortic coverage is identified as a variable that has a fundamental role in the development of SC ischaemia. Some authors identified a precise cut-off length (205 mm [32]), above which the aortic coverage length was showed to be an independent predictor of SCI. Almost the same covering length of $20 \mathrm{~cm}$ emerges from EUROSTAR data [5] as a threshold for other protocols that express the same aortic length. In these cases, a CSF drain should be placed between 12 and $20 \mathrm{~cm}$.

Some attempts to overstep the limit imposed by the endovascular approach have been made, from the successful creation of a type Ib endoleak to perfuse the lumbar vessels following the development of paraplegia (Reilly et al. [33] ) to the less successful incorporation of spinal perfusion branches in the endograft design (Ivanchev et al. [34]).

In recent years, in light of the "collateral network concept" (Furthermore, Etz et al. [35]), the staged repair is becoming a solid practice in the Crawford type II procedures, in an effort to maximize collateralization after the loss of elements of the collateral supply (Fig. 27.4).

O' Callaghan and Eagleton et al. (Cleveland Clinic, $\mathrm{OH}$ ) analysed their experience comparing single and staged Crawford type II TAAA procedures, with the result of a significantly lower overall rate of spinal injury $(37.5 \%$ vs $11 \%$ ) and mortality (Fig. 27.5). No optimal time interval between stages is described with certainty at present. The interval proposed in their analysis is determined by the 6- to 12-week delay in constructing a custom device, resulting in the median of 5 months. Two patients had rup-

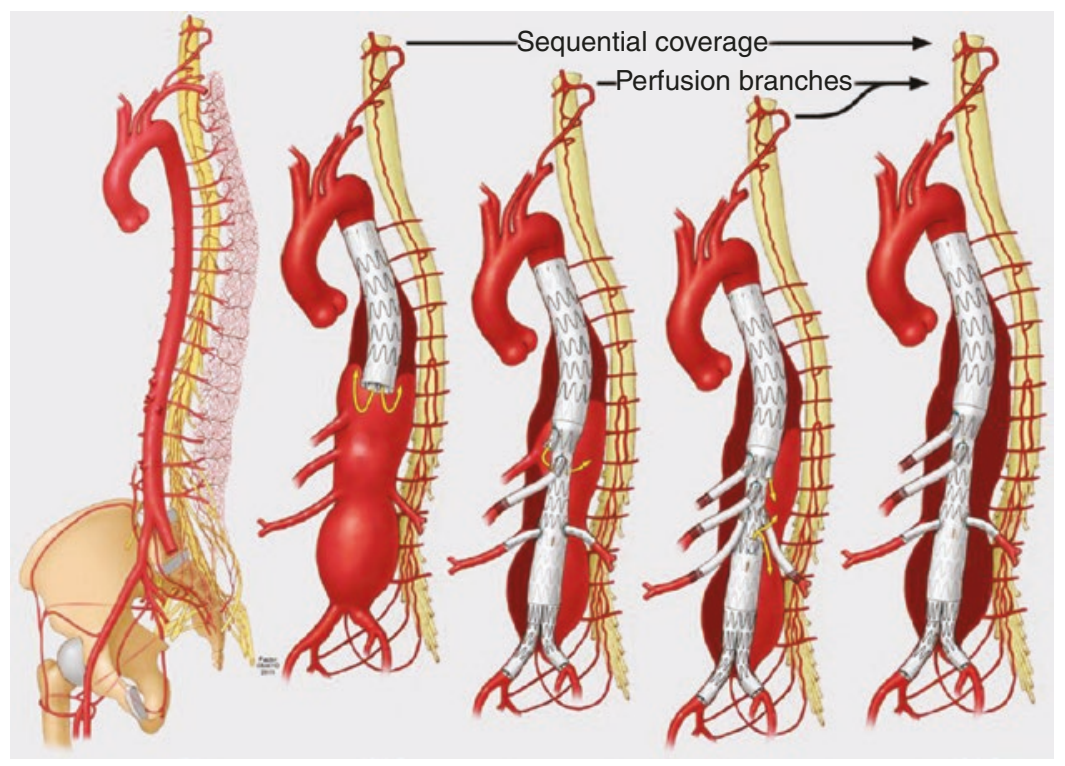

Fig. 27.4 Optimization of the extensive spine collateral network, including vertebral, intercostal, lumbar and hypogastric arteries, forms the basis for staged endovascular repair of complex aneurysms. Strategies include coverage of the proximal thoracic aorta up to the celiac axis, followed by visceral branch stenting in the second stage. Alternatively the sac can be perfused via perfusion branches or unstented celiac axis or contralateral iliac limb. The addition of two perfusion branches is favoured by some to improve haemodynamics and avoid hyperpressurization of the sac by single branches (Peter V. Banga, Gustavo S. Oderich, Leonardo Reis de Souza, Jan Hofer, Meaghan L. Cazares Gonzalez, Juan N. Pulido, Stephen Cha, Peter Gloviczki, Neuromonitoring, Cerebrospinal Fluid Drainage, and Selective Use of Iliofemoral Conduits to Minimize Risk of Spinal Cord Injury During Complex Endovascular Aortic Repair, Journal of Endovascular Therapy 2016, Vol. 23(1) 139-149) 
Fig. 27.5 KaplanMeier showing how the two-stage procedure affects patient survival

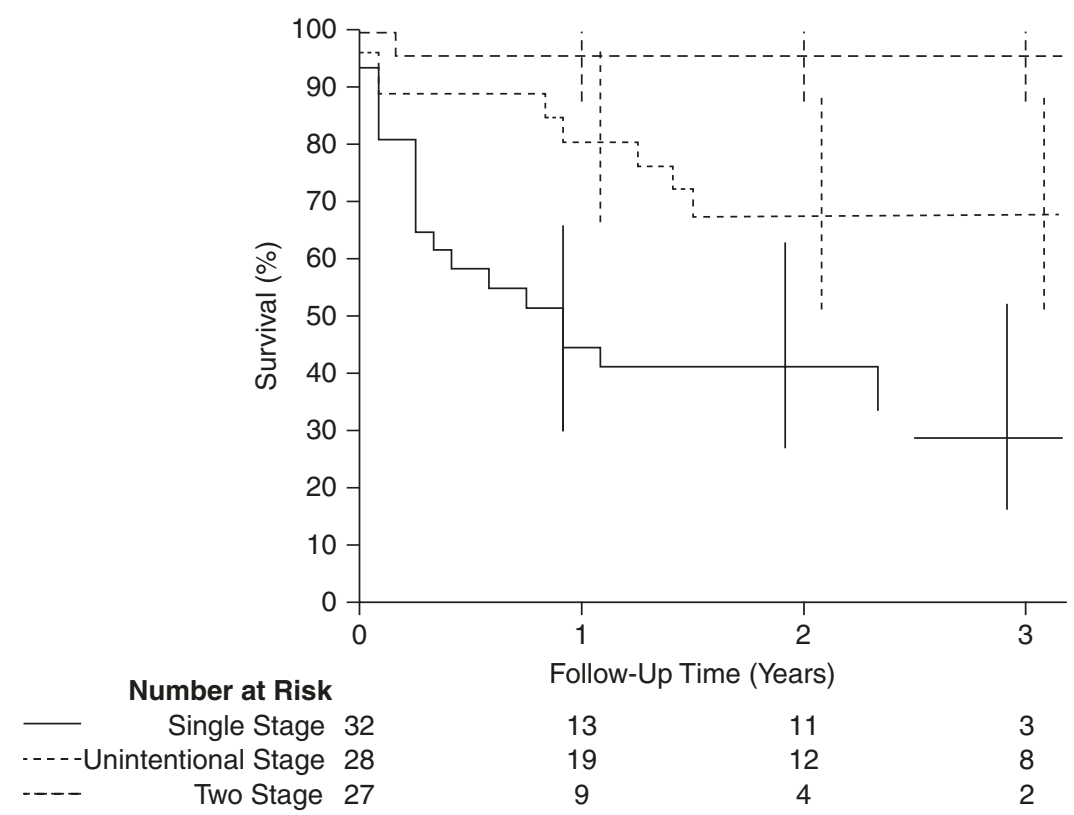

ture while they were waiting for completion of the second stage, so it is desirable to limit the time between stages in order to reduce the risk of rupture in the intervening period [36].

To maintain the intercostal and/or lumbar arteries patent after the first stage of endovascular aortic repair is at the base of the concept of temporary aneurysm sac perfusion (TASP). TASP consist of the noncompletion of at least one stent-graft side branch in order to prevent complete aneurysm sac thrombosis and maintain spinal cord perfusion through intercostal-lumbar arteries.

Kasprzak et al. studied the feasibility and early perioperative outcome of TAAAs treated with bEVAR compared with patients having bEVAR with immediate side branch. For TASP preferentially the celiac trunk was left noncompleted after uncomplicated superior mesenteric artery (SMA) and renal artery stent-graft implantation. Secondstage branch completion was recommended within 4 weeks of the initial bEVAR, and all patients with symptomatic TAAA had primary side branch completion. As a result they observed severe SCI in the non-TASP group immediately after the intervention in six patients and delayed in one patient versus a single paraplegia case immediately after bEVAR and one after the side branch completion in the TASP group.

The risk of aneurysm rupture during the TASP interval has to be considered, and based on their experience, early side branch completion within 4 weeks is recommended according to the presence or absence of symptoms of SCI [37].

\subsubsection{Minimally Invasive Segmental Artery Coil Embolization}

In order to overcome the need for staged procedures in the endovascular treatment of TAAA, the coil embolization strategy for intercostal segmental arteries (minimally invasive segmental artery coil embolization-MISACE) has been proposed.

As a result of the animal model studies [38], this technique, carried out through a percutaneous femoral approach, may allow extensive aortic repair in a single surgical time, increasing the resilience of spinal cord perfusion prior to definite aortic repair. 
Tilo Kolbel's group described the first-in-man (two patients) clinical application of endovascular coil embolization to precondition the spinal cord collateral network before open and totally endovascular extensive TAAA repair. After the identification of target spinal arteries (SAs), the first stage of the treatment consists of embolization of the SAs of the thoracic or abdominal level under local anaesthesia, aiming to occlude the vessels in their ostial segment to preserve the ability of the collateral network to compensate to a maximum extent $(2.7 \mathrm{~F}$ microcatheter and 0.018 microcoils). Endovascular coil embolization did not result in any neurologic impairment, and both patients were discharged after $72 \mathrm{~h}$ of clinical surveillance.

The interval between the MISACE and the second stage was $4-8$ weeks, although probably a shorter time may be sufficient to trigger the collateral network response, and the second procedures had a successful outcome [39].

Although minimally invasive segmental coil embolization seems feasible, risks and limitations need to be addressed to further optimize this technique.

\subsection{Operative Strategies for Preventing SCl in Open Repair}

During the clamp and sew era, aortic cross-clamp time was the principal predictor of neurologic impairment during thoracic and thoracoabdominal aortic repair. In Crawford's experience with 1509 patients, aortic cross-clamp time of more than 60 min was associated with a $27 \%$ incidence of paraplegia overall and $50 \%$ for the extent II TAAA [2].

A decade of study definitely refined a series of adjuncts, which includes distal aortic perfusion, selective intercostal artery reimplantation, cerebrospinal fluid drainage and neuromonitoring, that led to the separation of clamp time from ischaemic time when a multimodal SC prevention protocol is used.

In order to preserve the SC cord supply, solid preoperative planning with the study of "criti- cal" intercostal arteries must precede the surgical act; in addition to surgery, anaesthesiologic adjuncts can aim to increase SC tolerance to ischaemia, such as mild systemic hypothermia or deep hypothermic circulatory arrest, pharmacologic neuroprotection and controlled hypertension.

During open surgical aortic repair, three periods when the spinal cord is vulnerable to ischaemia are described: during aortic cross-clamping, after the clamp is removed if crucial spinal arteries are not perfused and, in some patients, for several days or weeks postoperatively [23].

\subsubsection{Intercostal Artery Reimplant}

While in the past authors advocated expeditious aortic repair with systemic sacrifice of intercostal arteries [40], with "the clamp and sew technique", being confident in the SC collateral network, at present, the role of preservation of spinal flow in reducing the risk of paraplegia/paraparesis is well known. The importance of the reattachment of critical intercostal arteries (T8-L2 zone) is widely demonstrated [41], and the procedure can be performed either systematically or selectively. The routine reimplant of all patent intercostal arteries in T7-L2 segment proved to reduce paraplegia rate below $10 \%$ in some series [42], but it can be time-consuming, and large "Carrel" patches may be susceptible to further dilatation. Therefore, it is questionable whether a large number of reimplanted intercostal arteries justify the effort and the risk for every patient; in particular it would probably be better to avoid unnecessary reimplantations especially in patients with connective tissue disorders. Interestingly, recent advances in CT and MR imaging technology may play a role in planning a selective reimplantation of the principal SC feeders [43].

The goal of selective reimplantation of the intercostal arteries is to overcome the disadvantages of systematic reimplantation, reducing the clamping time as well as the time of the entire procedure. 
ARM or some peculiar intercostal arteries that had potentially emerged at the preoperative imaging are certainly prone to selective reimplantation. Otherwise, intercostal arteries may be temporarily occluded during the procedure, and MEP/SSEP modification can guide intraoperative decisions. Only intercostal/lumbar arteries that lead to instrumental modification could potentially be reimplanted, preventing unnecessary intercostal artery reimplantation [44]. Otherwise intercostal arteries may even be sacrificed if MEP-SSEP monitoring remains unchanged.

Other authors advocate expeditious aortic repair with the systemic sacrifice of the intercostal arteries [40], "the clamp and sew technique", where the rationale is to avoid "the steal" phenomenon, sacrificing intercostal arteries and being confident in the spinal cord collateral network.

\subsubsection{Distal Aortic Perfusion}

The basic premise for distal aortic perfusion has been that increasing the distal aortic perfusion pressure will result in improved blood flow to the spinal cord and result in the benefit of decreased spinal cord ischaemic injury.

Retrograde distal aortic perfusion was imposed as a necessary prevention of spinal and visceral ischaemia when aortic clamping lasts longer than $30 \mathrm{~min}$. A number of approaches have been attempted for distal aortic perfusion and have included passive shunts, roller pumps, centrifugal pumps or total cardiopulmonary bypass. Passive pump shunts cannot regulate flow, while cardiopulmonary bypass needs significant systemic heparinization with the possibility of excessive bleeding. It has been our preference to use centrifugal pumps that have the advantage of avoiding or at least reducing heparinization, as well as the ability to control distal flow.

Coselli et al. reported a protective effect of the left heart bypass combined with sequential clamping compared with the simple "clamp and sew" technique. In his experience, patients treated with this adjunct had an incidence of SC ischaemia of $4.5 \%$ versus $11.2 \%$ in those with simple clamping technique, concluding that left heart bypass may play a crucial role in cases of unexpected complications or extensive repair [45].

\subsection{Postoperative SCI Prevention}

In the immediate postoperative period, it is mandatory to focus on a close neurological monitoring in order to put potential manoeuvers in place to minimize the risk of paraplegia at a very first sign of spinal cord ischaemia. Various protocols suggest different evaluation time and scales. One way to standardize the evaluation involves the use of muscle strength grading scale, for example, in assessing hip flexion and flexion and dorsiflexion of the foot.

The score is made as follows: 0 , no muscle contraction; 1 , contraction but no muscle shortening; 2 , with shortening but unable to overcome gravity; 3 , shortening able to overcome gravity, but no ability to gain additional strength; 4 , shortening, able to gain a small additional force; 5 , normal.

Different options have been explored for the management of CSF drainage: one option is to maintain or decrease the intraoperative target of drainage postoperatively for about 24-36 $\mathrm{h}$ and then clamp the drain following its removal at $48 \mathrm{~h}$, maintaining a MAP of $75-85 \mathrm{mmHg}$. Another approach is to suspend drainage postoperatively as soon as the patient is able to demonstrate normal force in the lower limbs, keeping MAP at $90-100 \mathrm{mmHg}$. If the patient develops symptoms, a neurological evaluation is recommended followed by haemodynamic optimization and CSF drainage increase (if it has been decreased in the meantime) [46].

Permissive hypertension (mean MAP of 90) is recommended, keeping haemoglobin $>9 \mathrm{mg} / \mathrm{dL}$ for the first 5 days post-op, and if the patient develops SCI, haemoglobin should be raised by $>10 \mathrm{mg} / \mathrm{dL}$ [3]. 


\subsection{Conclusions}

Spinal cord protection during open and endovascular aortic repair is still a major challenge. It is multifactorial and this underlines the need for a multimodal approach in the attempt to prevent it. The "aortic team" must involve surgeons, anaesthesiologists and intensive care specialists in order to put in place the best knowledge with the strongest efforts.

Spinal cord ischaemia is also time-dependent, forcing the team to act fast and resolutely. Focusing on that aspect, it may be auspicable to have bundled protocols with the rationale to facilitate guideline application by identifying key interventions from evidence-based guidelines or best clinical practices.

Even if recent studies and innovation have provided the surgeon with instruments for detecting early spinal cord injuries, an affordable and precise diagnostic tool does not yet exist. Further effort has to be made in that field.

At present, the state of the art in spinal cord protection can be resumed in an apparent contradictory sentence: try to implement multimodal bundled protocol in order to standardize surgical and rescue procedures, but at the same time remember to apply tailored techniques and procedures in order to fit each patient peculiarity.

\section{References}

1. Clopton MB. Surgery of the heart and blood vessels. A review of recent literature. 211 Clopton: Surgery of the heart. St. Louis Interstate Medical Journal Co, 1911.

2. Svensson LG, Crawford ES, Hess KR, Coselli JS, Safi HJ. Experience with 1509 patients undergoing thoracoabdominal aortic operations. J Vasc Surg. 1993;17:357-70.

3. Scali ST, Kim M, Kubilis P, Feezor RJ, Giles KA, Miller B, Fatima J, Huber TS, Berceli SA, Back M, Beck AW. Implementation of a bundled protocol significantly reduces risk of spinal cord ischemia after branched or fenestrated endovascular aortic repair. J Vasc Surg. 2017;67(2):409.e4-23.e4.

4. Bobadilla JL, Wynn M, Tefera G, Acher CW. Low incidence of paraplegia after thoracic endovascular aneurysm repair with proactive spinal cord protective protocols. J Vasc Surg. 2013;57:1537-42.
5. Buth J, Harris PL, Hobo R, van Eps R, Cuypers P, Duijm L, et al. Neurologic complications associated with endovascular repair of thoracic aortic pathology: incidence and risk factors. A study from the European Collaborators on Stent/Graft Techniques for Aortic Aneurysm Repair (EUROSTAR) registry. J Vasc Surg. 2007;46:1103-10. discussion: 1110-1.

6. Rossi SH, Patel A, Saha P, Gwozdz A, Salter R, Gkoutzios P, et al. Neuroprotective strategies can prevent permanent paraplegia in the majority of patients who develop spinal cord ischaemia after endovascular repair of thoracoabdominal aortic aneurysms. Eur J Vasc Endovasc Surg. 2015;50:599-607.

7. Schlosser FJ, Verhagen HJ, Lin PH, Verhoeven EL, van Herwaarden JA, Moll FL, et al. TEVAR following prior abdominal aortic aneurysm surgery: increased risk of neurological deficit. J Vasc Surg. 2009;49:30814. discussion: 314 .

8. Thron AK. Vascular anatomy of the spinal cord: neuroradiological investigations and clinical syndromes. New York: Springer; 1989.

9. Melissano G, Bertoglio L, Rinaldi E, Leopardi M, Chiesa R. An anatomical review of spinal cord blood supply. J Cardiovasc Surg. 2015;56(5):699-706.

10. Melissano G, Civilini E, Bertoglio L, Calliari F, Campos Moraes Amato A, Chiesa R. Angio-CT imaging of the spinal cord vascularisation: a pictorial essay. Eur J Vasc Endovasc Surg. 2010;39:436-40.

11. Adamkiewicz A. Die Blutgefasse des menschlichen Ruckenmarkes, I: Die Gefasse der Ruckenmarksubstanz. Sitzb Akad Wiss. 1881;84:469598; II Die Gefasse der Ruckenmarksoberflache. Sitzb Akad Wiss. 1882;85:101-30.

12. Kirklin JW, Barrat Boyes BG. Acute traumatic aortic transection. In: Cardiac surgery. New York: Wiley; 1986. p. 1462-4.

13. Skalski JH, Zembala M. Albert Wojciech Adamkiewicz: the discoverer of the variable vascularity of the spinal cord. Ann Thorac Surg. 2005;80:1971-5.

14. Kieffer E, Richard T, Chiras J, Godet G, Cormier E. Preoperative spinal cord arteriography in aneurysmal disease of the descending thoracic and thoracoabdominal aorta: preliminary results in 45 patients. Ann Vasc Surg. 1989;3:34e46.

15. Hyodoh H, Kawaharada N, Akiba H, Tamakawa M, Hyodoh K, Fukada J, et al. Usefulness of preoperative detection of artery of Adamkiewicz with dynamic contrast-enhanced MR angiography. Radiology. 2005;236(3):1004-9.

16. Svensson LG, Hess KR, Coselli JS, Safi HJ. Influence of segmental arteries, extent, and atriofemoral bypass on postoperative paraplegia after thoracoabdominal aortic operations. J Vasc Surg. 1994;20:255-62.

17. Kawaharada N, Morishita K, Fukada J, Yamada A, Muraki S, Hyodoh H, et al. Thoracoabdominal or descending aortic aneurysm repair after preoperative demonstration of the Adamkiewicz artery by magnetic resonance angiography. Eur $\mathrm{J}$ Cardiothorac Surg. 2002;21(6):970-4. 
18. Chiesa R, Melissano G, Marrocco-Trischitta MM, Civilini E, Setacci F. Spinal cord ischemia after elective stent-graft repair of the thoracic aorta. J Vasc Surg. 2005;42:11-7.

19. Setacci F, Sirignano P, Cappelli A, Setacci C. The wonders of a newly available post-analysis ct software in the hands of vascular surgeons. Eur J Vasc Endovasc Surg. 2012;43:404-6.

20. Melissano G, Bertoglio L, Civelli V, Moraes Amato AC, Coppi G, Civilini E, Calori G, De Cobelli F, Del Maschio A, Chiesa R. Demonstration of the Adamkiewicz artery by multidetector computed tomography angiography analysed with the opensource Software OsiriX. Eur J Vasc Endovasc Surg. 2009;37:395-400.

21. Czerny M, Eggebrecht H, Sodeck G, Verzini F, Cao P, Maritati G, Riambau V, Beyersdorf F, Rylski B, Funovics M, Loewe C, Schmidli J, Tozzi P, Weigang E, Kuratani T, Livi U, Esposito G, Trimarchi S, van den Berg JC, Weiguo F, Chiesa R, Melissano G, Bertoglio L, Lonn L, Schuster I, Grimm M. Mechanisms of symptomatic spinal cord ischemia after TEVAR: insights from the European registry of endovascular aortic repair complications (EuREC). J Endovasc Ther. 2012;19:37-43.

22. Eagleton MJ, Shah S, Petkosevek D, Mastracci TM, Greenberg RK. Hypogastric and subclavian artery patency affects onset and recovery of spinal cord ischemia associated with aortic endografting. J Vasc Surg. 2014;59:89-95.

23. Godet G, Bertrand M, Fléron M-H, Goarin J-P, Colson P, Cardon A, Koskas F, Verhoye J-P, Kieffer E. Cerebrospinal fluid drainage and thoracic endovascular aneurysm repair. Asian Cardiovasc Thorac Ann. 2017;25(9):608-17.

24. Sugie S, Nakajima M, Ueno A, et al. Studies on occlusion and replacement of the thoracic aorta. Nippon J Angiocardiol. 1957;20:120.

25. McCullough JL, Hollier LH, Nugent M. Paraplegia after thoracic aortic occlusion: influence of cerebrospinal fluid drainage. Experimental and early clinical results. J Vasc Surg. 1988;7:153-60.

26. Bower TC, Murray MJ, Gloviczki P, et al. Effects of thoracic aortic occlusion and cerebrospinal fluid drainage on regional spinal cord blood flow in dogs: correlation with neurologic outcome. J Vasc Surg. 1989;9:135-44.

27. Tshomba Y, Leopardi M, Mascia D, Kahlberg A, Carozzo A, Magrin S, Melissano G, Chiesa R. Automated pressure-controlled cerebrospinal fluid drainage during open thoracoabdominal aortic aneurysm repair. J Vasc Surg. 2017;66:37-44.

28. Coles JG, Wilson GJ, Sima AF, et al. Intraoperative detection of spinal cord ischemia using somatosensory cortical evoked potentials during thoracic aortic occlusion. Ann Thorac Surg. 1982;34(3):299-306.

29. Cunnigham JN Jr, Lashinger JC, Spencer FC. Monitoring of somatosensory evoked potentials during surgical procedures on the thoracoabdominal aorta. IV. Clinical observations and results. J Thorac Cardiovasc Surg. 1987;94(2):275-85.

30. Laschinger JC, Cunnigham JN Jr, Cooper MM, et al. Monitoring of somatosensory evoked potentials during surgical procedures on the thoracoabdominal aorta. I. Relationship of aortic cross-clamp duration, changes in somatosensory evoked potentials, and incidence of neurologic dysfunction. J Thorac Cardiovasc Surg. 1987;94(2):260-5.

31. Banga PV, Oderich GS, de Souza LR, Hofer J, Cazares Gonzalez ML, Pulido JN, Cha S, Gloviczki P. Neuromonitoring. Cerebrospinal fluid drainage, and selective use of iliofemoral conduits to minimize risk of spinal cord injury during complex endovascular aortic repair. J Endovasc Ther. 2016;23(1):139-49.

32. Amabile P, Grisoli D, Giorgi R, et al. Incidence and determinants of spinal cord ischaemia in stent-graft repair of the thoracic aorta. Eur J Vasc Endovasc Surg. 2008;35:455-61.

33. Reilly LM, Chuter TA. Reversal of fortune: induced endoleak to resolve neurological deficit after endovascular repair of thoracoabdominal aortic aneurysm. J Endovasc Ther. 2010;17:21-9.

34. Harrison SC, Agu O, Harris PL, Ivancev K. Elective sac perfusion to reduce the risk of neurologic events following endovascular repair of thoracoabdominal aneurysms. J Vasc Surg. 2012;55:1202-5.

35. Etz CD, Kari FA, Mueller CS, Brenner RM, Lin HM, Griepp RB. The collateral network concept: remodeling of the arterial collateral network after experimental segmental artery sacrifice. J Thorac Cardiovasc Surg. 2011;141:1029-36.

36. O'Callaghan A, Mastracci TM, Eagleton MJ. Staged endovascular repair of thoracoabdominal aortic aneurysms limits incidence and severity of spinal cord ischemia. J Vasc Surg. 2015;61:347-54.

37. Kasprzak PM, Gallis K, Cucuruz B, Pfister K, Janotta M, Kopp R. Editor's choice-temporary aneurysm sac perfusion as an adjunct for prevention of spinal cord ischemia after branched endovascular repair of thoracoabdominal aneurysms. Eur J Vasc Endovasc Surg. 2014;48(3):258-65.

38. Luehr M, Salameh A, Haunschild J, et al. Minimally invasive segmental artery coil embolization for preconditioning of the spinal cord collateral network before one stage descending and thoracoabdominal aneurysm repair. Innovations. 2014;9: $60-5$.

39. Christian D, Sebastian Debus E, Moh F-W, Kolbel T. First-in-man endovascular preconditioning of the paraspinal collateral network by segmental artery coil embolization to prevent ischemic spinal cord injury. J Thorac Cardiovasc Surg. 2015;149(4): 1074-9.

40. Galla JD, Ergin MA, Lansman SL, McCullough JN, Nguyen KH, Spielvogel D, Klein JJ, Griepp RB. Use of somatosensory evoked potentials for thoracic and thoracoabdominal aortic resections. Ann Thorac Surg. 1999;67(6):1947-52. discussion 1953-8. 
41. Safi HJ, Miller CC, Carr C, Iliopoulos DC, Dorsay DA, Baldwin JC. Importance of intercostal artery reattachment during thoracoabdominal aortic aneurysm repair. J Vasc Surg. 1998;27(1):58-66. discussion 66-8.

42. Jacobs MJ, de Mol BA, Elenbaas T, Mess WH, Kalkman CJ, Schurink GW, Mochtar B. Spinal cord blood supply in patients with thoracoabdominal aortic aneurysms. J Vasc Surg. 2002;35(1):30-7.

43. Melissano G, Bertoglio L, Mascia D, Rinaldi E, Del Carro U, Nardelli P, Chiesa R. Spinal cord ischemia is multifactorial: what is the best protocol? J Cardiovasc Surg. 2016;57(2):191-201.

44. Davidovic L, Ilic N. Spinal cord ischemia after aortic surgery. J Cardiovasc Surg. 2014;55(6):741-57.

45. Coselli JS. The use of left heart bypass in the repair of thoracoabdominal aortic aneurysms: current techniques and results. Semin Thorac Cardiovasc Surg. 2003;15(4):326-32.

46. Dias-Neto M, Reis PV, Rolim D, Ramos J'F, Teixeira J'F, Sampaio S. Strategies to prevent TEVAR-related spinal cord ischemia. Vascular. 2017;25(3):307-15. 